\title{
Evaluation of Text Mining Techniques Using Twitter Data for Hurricane Disaster Resilience
}

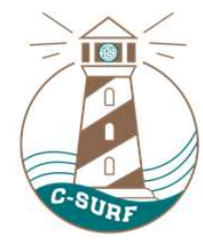

Research Objectives

1. Analyze and evaluate Latent Dirichlet Allocation (LDA) topic model performance.

2. Analyze semantic interpretation, sentiment, and volume of tweets surrounding disasters.

\begin{tabular}{|l|}
\multicolumn{1}{|c|}{ Introduction } \\
- Text mining has been used to model public \\
opinion and sentiment about various topics \\
- Latent Dirichlet Allocation (LDA) topic \\
models have limitations when used to model \\
data collected from Twitter \\
- Terms in topic/word \\
- Sentiment and volume analysis can provide \\
human intelligence to emergency managers \\
\hline
\end{tabular}

\begin{tabular}{|l|}
\multicolumn{1}{c|}{ Data } \\
Collection \\
- $2,728,730$ tweets collected from Twitter \\
Stream API \\
- Location: North Carolina \& South Carolina \\
- Sept 1 - Oct 1, 2018 (Hurricane Florence) \\
\multicolumn{1}{c|}{ Preprocessing } \\
- Dataset was reverse geotagged \\
- Keyword searched and filtered using 31 \\
hurricane-related terms \\
- Duplicate tweets removed \\
- Datasets created for experiments \\
Experimental Methodology \\
Latent Dirichlet Allocation Experimentation \\
- Coherence experimentation 120 models \\
- $5-60$ topics, 5 pooling methods, 2 stemming \\
methods \\
- Semantic interpretation (qualitative) \\
Sentiment/Volume Analysis \\
- Geospatial (state-level) analysis \\
- Temporal analysis \\
- Responses to specific events \\
\hline
\end{tabular}

Creighton

College of Arts and Sciences
Joshua Eason ${ }^{1}$, Sathish Kumar ${ }^{2}$

${ }^{1}$ Dept. of Physics, Creighton University

${ }^{2}$ Dept. of Computing Sciences, Coastal Carolina University

josheason@Creighton.edu

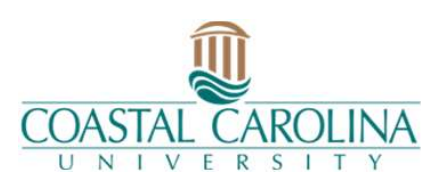

\section{Results}

LDA Model Performance Evaluation

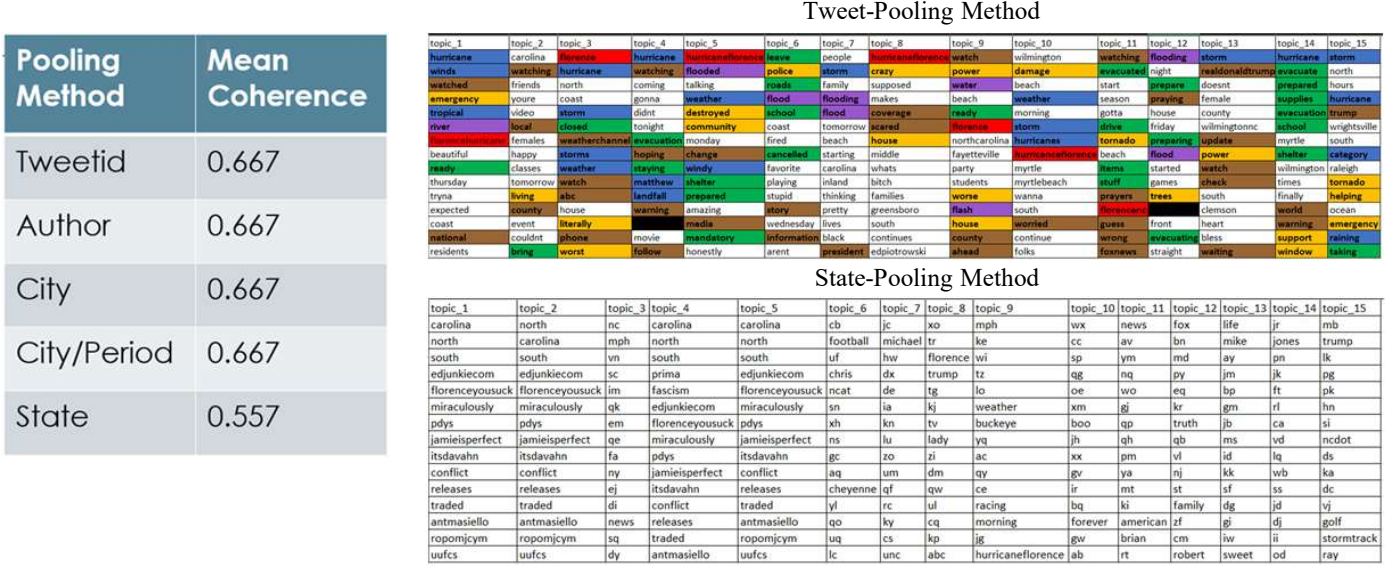

\section{Sentiment/Volume Analysis}
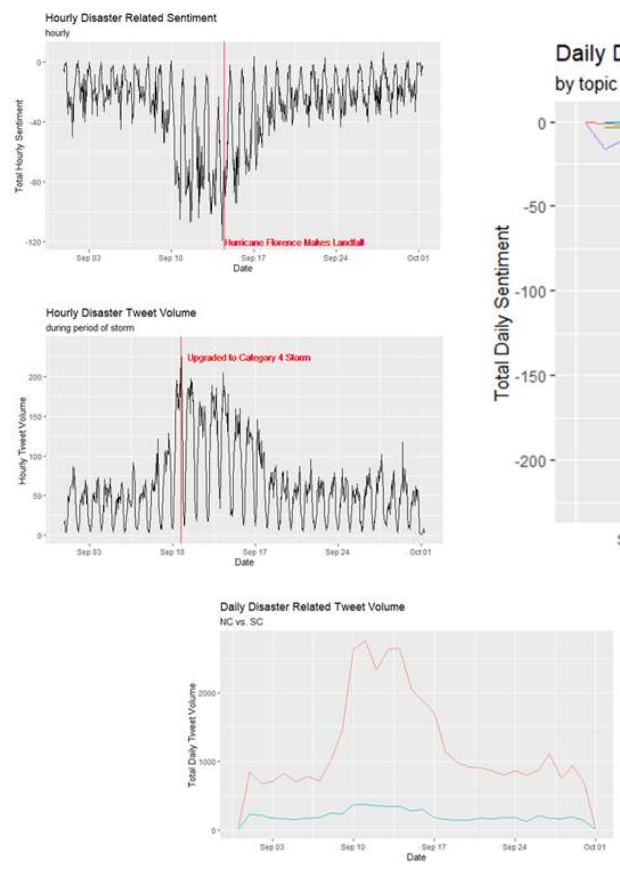

Daily Disaster Related Tweet Total Sentiment

$$
\text { by topic }
$$

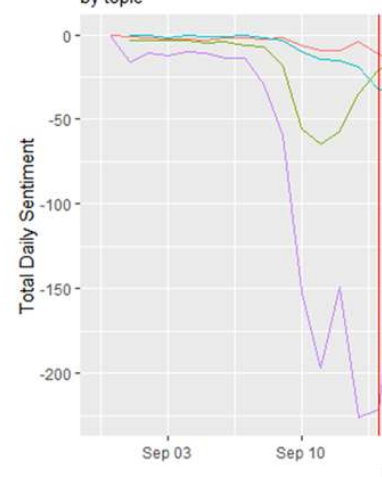

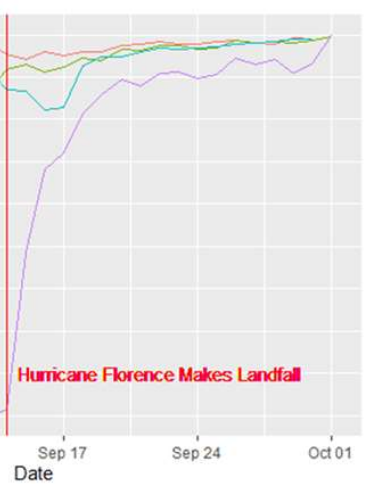

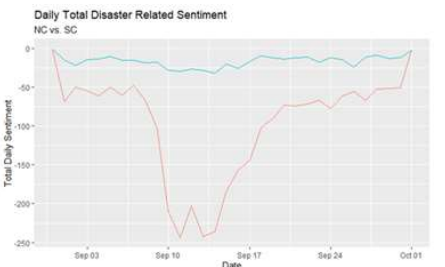

aftermath

- evacuation

hurricane

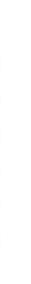

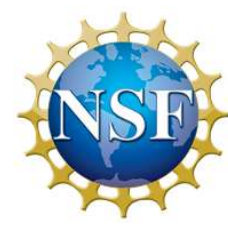

Acknowledgments

- Funding for C-SURF was provided by NSF REU Award AGS 1560210

Dr. Zhenlong Li, Department of Geography, University of South Carolina, Columbia

Michael Bunker, Department of Computing Sciences, Coastal Carolina University 